\title{
BMC Medical Ethics reviewer acknowledgement 2015
}

Clare Partridge

\section{Contributing reviewers}

The editors of BMC Medical Ethics would like to thank all our reviewers who have contributed to the journal in Volume 16 (2015).

Beyene Ademe

Ethiopia

Kaoruko Aita

Japan

Aslihan Akpinar

Turkey

Eman Al Gaai

Saudi Arabia

Ghiath Alahmad

Saudi Arabia

Afshan Ali

Saudi Arabia

James A Anderson

Canada

Armand Antommaria

USA

John Appleby

UK

Kavita Shah Arora

USA

Atsushi Asai

Japan

Richard Ashcroft

UK

Marc Avey

Canada
Rahime Aydin Er

Turkey

Gabriele Badano

UK

Kristine Bærøe

Norway

Michael Balboni

USA

Francis Barchi

USA

Jo Barnes

New Zealand

Bruce Barron

USA

Steve Bellan

USA

Solomon (Solly) Benatar

South Africa

Rebecca Bennett

UK

Hazel Biggs

UK

Giles Birchley

UK

Isra Black

UK

\section{Jonathan Boote \\ UK}

Kirstin Borgerson

Canada

Elaine Boyle

UK

\section{Iain Brassington}

UK

Anders Bremer

Sweden

Berit Bringedal

Norway

Arianne Brinkman-Stoppelenburg Netherlands

Rebecca Brown

UK

Teneille Brown

USA

\section{Susan Bull}

UK

Emma Bullock

Hungary

Luciana Caenazzo

Italy

Megan Campbell

South Africa 
Emma Cave

UK

David Chambers

USA

Tracey Chantler

UK

Phaikyeong Cheah

Thailand

Y.Y. Brandon Chen

Canada

Yen-Yuan Chen

Taiwan

Jeannie Cimiotti

USA

John Coggon

UK

Gilberto Corbellini

Italy

Amy Corneli

USA

Anselm Crombach

Germany

Andrew Crowden

Australia

Sylvia Cruess

Canada

Luis Gabriel Cuervo

USA

Barbara Daly

USA

Myriam Deveugele

Belgium

Veljko Dubljevic

Canada

Marleen Eijkholt

USA

Carolyn Ells

Canada

Jakob Elster

Norway

Nathan Emmerich

UK

Stefan Eriksson

Sweden
Anna Falcó-Pegueroles

Spain

Zaher Fanari

USA

Anne-Maree Farrell

Australia

Michael Fay

UK

Richard Fielding

Hong Kong

Kjersti Fjørtoft

Norway

Paul Ford

USA

Nikolaus Forgo

Germany

Veronique Fournier

France

Sara Fovargue

UK

Allessandra Gasparetto

Italy

Joel Geiderman

USA

Eric Geijteman

Netherlands

Paul Wenzel Geissler

Norway

Davina Ghersi

Australia

David Gibson

UK

Noor Giesbertz

Netherlands

Simona Giordano

UK

Nada Gligorov

USA

Trudy Goodenough

UK

Christine Grady

USA

Gilly Griffin

Canada
Danielle Griffiths

UK

Marilys Guillemin

Australia

Freedom Nkhululeko Gumedze South Africa

Karen Gutierrez

USA

Akiko Hagimoto

Japan

Richard Hain

UK

Daniel Hall

USA

Sumaya Hammami

USA

Thor Willy Ruud Hansen

Norway

Lawrence Hansen

USA

Bhakti Hansoti

USA

Lena Hedén

Sweden

Irma Hein

Netherlands

Gert Helgesson

Sweden

Matthew Herder

Canada

Elizabeth Hill

UK

Ming-Jung Ho

Taiwan

Bjorn Hofmann

Norway

Anna Höglund

Sweden

Ruth Horn

UK

Samia Hurst

Switzerland

Adam Hutchings

UK 
Dawn Ionescu

USA

Jonathan Ives

UK

Nichon Jansen

Netherlands

Rien Janssens

Netherlands

Steven Joffe

USA

Franz Johnson

USA

Niklas Juth

Sweden

Jaranit Kaewkungwal

Thailand

Michael Kalichman

USA

Dorcas Kamuya

Kenya

Daniel Kim

USA

Jonathan Kimmelman

Canada

Celia Kitzinger

UK

Satoshi Kodama

Japan

Erwin Kompanje

Netherlands

Elleke Landeweer

Netherlands

Adele Langlois

UK

Graeme Laurie

UK

Karen Le Ball

UK

Brian Lee

USA

Aaron Levine

USA

Penney Lewis

UK
Charles Lidz

USA

Graham Lindegger

South Africa

Bebe Loff

Australia

Geir Lorem

Norway

Sana Loue

USA

Michael Loughlin

UK

Pekka Louhiala

Finland

Anneke Lucassen

UK

Andreas Lundh

Denmark

Jeroen Luyten

UK

\section{Alan Lyles}

USA

Niels Lynöe

Sweden

Malcolm Macleod

UK

Morten Magelssen

Norway

Barry Main

UK

Kathryn Maitland

UK

Mario Malicki

Croatia

Phillipa Malpas

New Zealand

Manassé Bambonyé

Burundi

Vicki Marsh

Kenya

Patricia Marshall

USA

Wayne Martin

UK
Fred Martineau

UK

Zubin Master

USA

Keikantse Matlhagela

Botswana

Doreen Matsui

Canada

Pauline McCormack

UK

Fiona McDermott

Australia

Alex McKeown

UK

Marcel Mertz

Germany

Kenneth Miller

USA

Bert Molewijk

Netherlands

Keymanthri Moodley

South Africa

Marilyn Morris

USA

Solange Nappo

Brazil

Darcia Narvaez

USA

Oktawian Nawrot

Poland

Joseph Ochieng

Uganda

Gearoid O'Cuinn

UK

Anke Oerlemans

UK

Dermot O'Reilly

UK

Dominik Ose

Germany

Gareth Owen

UK

John Owens

UK 
Michael Parker

UK

Sarah Parsons

UK

Andy Petros

UK

Bernhard Pommer

Austria

Rouven Porz

Switzerland

Muireann Quigley

UK

Leah Rand

UK

Samiran Ray

UK

Sabi Redwood

UK

Dean Regier

Canada

Massimo Reichlin

Italy

Stuart Rennie

USA

David Resnik

USA

Solina Richter

Canada

Blanca Rodriguez Lopez

Spain

David Rodriguez-Arias

Spain

Wolf Rogowski

Germany

Philip Rosoff

USA

Michael Rowe

USA

Sabine Salloch

Germany

Lars Sandman

Sweden

Silke Schicktanz

Germany
Jan Schildmann

Germany

Sebastian Schleidgen

Germany

Harald Schmidt

USA

Guy Schofield

UK

Mark Schweda

Germany

Donnie Self

USA

Daniel Sessler

USA

Jane Seymour

UK

Anne Slowther

UK

Jeremy Snyder

Canada

Margaret Somerville

Canada

Antonio G Spagnolo

Italy

Dominique Sprumont

Switzerland

Shalini Sri Ranganathan

Sri Lanka

Ruth Stirton

UK

Lynne Stobbart

UK

John Stone

USA

Tuija Takala

Finland

Kate Tan

Australia

Attila Tanyi

UK

James Thomas

USA

Paulina Tindana

Ghana
Manuel Trachsel

Switzerland

Krista Tromp

Netherlands

Mark Tschaepe

USA

Edith Valdez-Martinez

Mexico

Rieke van der Graaf

Netherlands

Robert Van Howe

USA

Werdie van Staden

South Africa

Ghislaine van Thiel

Netherlands

Liliana Varesco

Italy

Dorothy Vawter

USA

Joseph Verheijde

USA

Giuseppe Vertrugno

Italy

Alice Virani

Canada

Polychronis Voultsos

Greece

Katherine Wade

Ireland

Julia Wade

UK

Elizabeth Wager

UK

Susan E Wallace

UK

Nancy Walton

Canada

Douglas Wassenaar

South Africa

Michael Weaver

USA

Guy Widdershoven

Netherlands 
Claudia Wiesemann

Germany

Verina Wild

Switzerland

Stephen Wilkinson

UK

Garrath Williams

UK
Eva Winkler

Germany

Jean Woo

Hong Kong

Julie Woodley

UK

Simon Woods

UK
Bridget Young

UK

Matjaz Zwitter

Slovenia 